\section{Wild boar injuries in hunting dogs: A retrospective study (2012-2016)}

\section{Panagiotis Kokkinos ${ }^{1}$, Demetris Tselekis ${ }^{2}$, Georgios Kazakos ${ }^{3}$, Lysimachos Papazoglou ${ }^{3}$, loannis Tsokatarides ${ }^{4}$}

1 Animal Health Trust, Lanwades Park, Kentford, Newmarket, Suffolk CB8 7UU, United Kingdom

2 Private Practice, Sparti, Greece

3 Companion Animal Clinic, School of Veterinary Medicine, Aristotle University of Thessaloniki, Thessaloniki, Greece

4 Private Practice, Drama, Greece

\section{OBJECTIVES}

Description of signalment, wound characteristics, treatment and outcome of wild boar injuries in hunting dogs.

\section{METHODS}

In this retrospective study, medical records of hunting dogs from a first opinion and a referral setting were reviewed for wild boar injuries. Data for 117 dogs were collected and included signalment, physical examination findings, wound characteristics, season of injury, boar sex, type of treatment and outcome among others.

\section{RESULTS}

The majority of both the injured dogs ( $82 \%$ ) and the boars (85\%) were males. Over three quarters of dogs $(77 \%)$ had a prior history of wild boar injury. The incidents occurred more frequently in December (30\%). The most common site of injury was the abdomen (43.6\%), followed by the hindlimbs (31.6\%) and the thorax (21.4\%), whereas $44 \%$ suffered multiple injuries. Wound exploration was indicated in $64 \%$ of abdominal and $72 \%$ of thoracic injuries. Pneumothorax and shock were seen in $20 \%$ and $22.4 \%$ of dogs, respectively. Surgical treatment was undertaken in $95.6 \%$ of the cases. The mean hospitalization time was 4.65 days. The outcome in $95.6 \%$ of the cases was good; moreover, $99 \%$ of the dogs returned to hunting activity in 26.3 days on average.

\section{STATEMENT (CONCLUSIONS)}

The confliction of hunting dogs with wild boars resulted in severe and extended injuries in dogs, usually requiring surgical intervention. Nevertheless, most of the dogs had a good outcome and returned to hunting activity after recovery period. To the authors' knowledge this is the first descriptive study about wild boar injuries in hunting dogs.
Results of artificial urethral sphincter placement in female dogs with refractory urinary incontinence

\section{Joep Timmermans ${ }^{1}$, Bart Van Goethem ${ }^{1}$, Bouvien Brocks ${ }^{2}$, Hilde De Rooster ${ }^{1}$}

1 Ghent University, Merelbeke, Belgium

2 Anicura Dierenkliniek Eersel, Eersel, Netherlands

\section{OBJECTIVES}

To determine if placement of an artificial urethral sphincter provides continence in female dogs with refractory urinary incontinence.

\section{METHODS}

A retrospective analysis of multi-institutional patient databases resulted in 15 female dogs with refractory urinary incontinence without concurrent underlying disease, treated between September 2010 and March 2018 A hydraulic artificial urethral sphincter with subcutaneous access port was placed according to the manufacturer's instructions. Treatment success was evaluated using a continence scoring system (scale 1 to 10 , complete incontinence to complete continence, respectively) through telephone enquiry.

\section{RESULTS}

Median continence score preoperatively was 2/10 (range, 1 to 5). Median follow-up was 6 months (range, 3 months to 5,8 years). Postoperative percutaneous cuff inflation was performed in $7 / 15$ dogs (47\%). Complete continence was achieved in $8 / 15$ dogs (53\%), improvement in $4 / 15$ dogs $(27 \%)$, no effect in $2 / 15$ dogs $(13 \%)$, and worsening in $1 / 15$ dogs $(7 \%)$. In the patients that improved but were not cured postoperatively, additional medical therapy resulted in a postoperative continence score of ${ }^{3} 9 / 10$ in $2 / 5$ dogs. Major complications necessitating implant removal surgery occurred in $3 / 15$ dogs $(20 \%)$, minor complications occurred in $10 / 15$ dogs (67\%). Continence scores were awarded to $10 / 15$ dogs (67\%). Median continence score postoperatively at follow-up was 9.5/10 (range, 9 to 10).

\section{STATEMENT (CONCLUSIONS)}

The artificial urethral sphincter provides promising results in the treatment of bitches with refractory incontinence. In dogs that improve but are not cured after artificial urethral sphincter placement, supplementary medical therapy can further improve the continence rate. 